\begin{tabular}{l} 
BENTHAM OPEN \\
CrossMark \\
Content list available at: www.benthamopen.com/TOBIOTJ/ \\
DOI: $10.2174 / 1874070701610010287$ \\
\hline
\end{tabular}

EDITORIAL

\title{
Microbial Biotechnology and Environmental Bioremediation: Challenges and Prospects
}

Humankind has seen tremendous progress in the last century in different industrial sectors. Industries such as, mining, health and medicine (especially therapeutics-antibiotics), textiles, fertilizers, pesticides, petrochemicals and refineries, are amongst the many others that advanced due to scientific progress. But such rapid progress has also led to elevateduncontrolled discharge of highly toxic chemicals and intermediates, which contribute to soil, water and air pollution. In general, nature takes care of recycling the resources and thus remediating the pollution related to soil, water and air. However, in the recent past, we have been witnessing pollution - beyond natural healing. Apart from industrialization, some of the other reasons are increase in population, over-exploiting the natural resources, deforestation, erosion of fertile soils, accidental-intentional release of hydrocarbons in the sea and land (oil spills), usage of chemical compounds like pesticides, indiscriminate use of antibiotics (as a food additives in meat industries, uncontrolled use of antibiotics in medicine field, etc.) and so on. Microbial biotechnology offers environmentally friendly approaches that can be implemented effectively for identifying the indicator microbial strains - causative agent for the problem and the ones useful for environmental bioremediation. The aspects of microbial-driven bioremediation process can benefit the community by exploiting the metabolomics capabilities of microorganisms that enable the utilization of toxic compounds and biotransformation to utilizable intermediates and products. While the conversion of agro-industrial waste products or municipal waste water to industrially useful value added products has already been exploited as an attractive option. It is generally expected that the candidate microbe and the metabolite intended for bioremediation should be non-pathogenic, non-toxic, economically and commercially viable, active and stable under harsh environmental conditions. Recent advancements in synthetic-biology, OMICS, and genetic engineering tools pave the way to achieve those selective criteria, identifying the indicator strains, route of pollution and development of tailormade microbe-metabolites that can be effectively applied for the environmental bioremediation.

The thematic issue "Microbial Biotechnology and Environmental Bioremediation: Challenges and Prospects" through research articles and reviews written by different biotechnologists and experts in their field offers a glimpse at recent findings on antibiotic resistance and indicator microorganisms, heavy metal pollution and applications related to resistant microbes, polycyclic aromatic hydrocarbons (PAHs) and oil pollution bioremediation, enzymes for bioremediation, and the production of useful bioproducts from toxic-waste materials. The special issue consists of 9 research articles/reviews contributed by authors from Oman, India, the USA and the UK.

Antibiotic resistant and multiple-antibiotic resistant bacteria (MARB) have become widespread issue, and one of the main reasons is the overuse (abuse) of antibiotics in therapeutics and in growth promotion for livestock. This demoralizes the usefulness of the drugs and mounts a serious crisis for human health. The contribution by Paulson et al., highlights the problem, and proposes that the level of antibiotic-resistant bacteria in wildlife environment could be used as a 'bio-indicator', to monitor pollution and to evaluate the success of efforts to curtail it. Second research paper by Al-Bahry et al., aims at demonstrating the diverse tetracycline resistant genes in Escherichia coli, as an indicator bacteria, isolated from different human and environmental sources. They used molecular biology tools and reported 4 'tet gene-combination' for the first time from their Middle Eastern region. They also stressed that the increase in the resistance of tetracycline with high diversification is an indication of antibiotic's overuse in the region.

The review by Al-Musharafi, aims at providing the summary of recent studies from arid regions on heavy metals pollution in sewage treated effluents and microbial remediation as a potential method. She further highlights the need of 
stricter industrial regulatory standards to be established and enforced to regulate the spread of non-essential metals in the environment. Al-Battashi et al., reviews the geo-microbiology of chromium $(\mathrm{Cr})$ pollution and the recent studies on the microbial diversity of $\mathrm{Cr}$-resistant microorganisms and their potential in the removal of $\mathrm{Cr}$ from the contaminated environmental sources.

Hydrocarbon contamination of soil and water is one of the major environmental problems, mainly linked to petroleum industries. Gupte et al., reviews the environmental issues pertaining to PAHs in the environment, its structures, environmental fate and toxicity issues, as well as different cost-effective bioremediation techniques. The review by AlSayegh et al., attempts to summarize the issues related to crude oil contamination and its biodegradation through bacteria by using it as carbon source. They also highlighted bacterial upgrading of heavy crude to lighter fractions and to aid its recovery. Chhaya and Ingale review the chemistry of micellar enzymology and its probable applications in bioremediation of chloro-phenolic environmental pollutants.

Utilization of different toxic and waste products can be an alternative to curb environmental pollution and at the same time, industrial production of useful bioproducts. Ramkumar et al., reviews the potential of fish-waste as a low cost substrate for the production of protease enzyme by bacteria. Whereas, Greenwell et al. highlighted simultaneous biosurfactant production and biodegradation of leather dust waste from tannery, in a research article.

The guest editor firmly believes that the collection of papers in this special issue will be of interest and useful to the research community.

\section{CONFLICT OF INTREST}

The author confirms that this article content has no conflict of interest.

\section{ACKNOWLEDGEMENTS}

I wish to thanks all the authors for their contribution and especially to all the reviewers for their critical insight and timely reviews of the manuscripts, without which this thematic issue was not possible. I would also like to thank my organization, Sultan Qaboos University, for encouragement and support.

Sanket J. Joshi

Central Analytical and Applied Research Unit

College of Science

Sultan Qaboos University

Oman

E-mail: sanket@squ.edu.om/sanketjj@gmail.com 